\title{
Safety and clinical efficacy of everolimus in the treatment of advanced renal cell carcinoma (RCC)
}

This article was published in the following Dove Press journal:

Drug, Healthcare and Patient Safety

25 June 2010

Number of times this article has been viewed

\section{Rohan Shahani \\ Kevin G Kwan \\ Anil Kapoor}

Division of Urology, Department of Surgery, St. Joseph's Healthcare Hamilton and McMaster University, Hamilton, Ontario, Canada
Correspondence: Anil Kapoor Associate Professor, Doug and June Barber Chair in Renal Disease, Division of Urology, McMaster University, McMaster Institute of Urology at St. Joseph's Healthcare, 50 Charlton Ave., E Hamilton, ON L8N 4A6, Canada Tel + I (905) 5226536

$\mathrm{Fax}+\mathrm{I}(905) 5216195$

Email akapoor@mcmaster.ca
Abstract: Renal cell carcinoma (RCC) is one of the most lethal genitourinary malignancies. Recently, there has been a paradigm shift in the management of advanced RCC. New targeted therapies including vascular endothelial growth factor (VEGF) and mammalian target of rapamycin (mTOR) inhibitors have been developed which have shown promising results in a patient population who otherwise had very few options for treatment. The first mTOR inhibitor, temsirolimus, an intravenous prodrug, has shown improved overall survival in poor prognosis patients. More recently, an oral mTOR inhibitor, everolimus (RAD 001), has been developed which has been shown to delay disease progression in patients with metastatic RCC who have progressed on other targeted therapies. Although a survival advantage in phase III trials is seen with everolimus, associated systemic toxicities, while generally well tolerated, are not insignificant. These include mucositis, hyperglycemia, hyperlipidemia, and pneumonitis. Despite the side effects, emerging evidence points to everolimus as the optimal second-line treatment for patients with advanced renal cell carcinoma.

Keywords: metastatic renal cell carcinoma, everolimus, mTOR inhibitors, VEGF inhibitors

\section{Epidemiology of renal cell carcinoma}

Renal cell carcinoma, which accounts for $2 \%$ to $3 \%$ of all adult malignant neoplasms, is the most lethal of all genitourinary malignancies. ${ }^{1}$ Traditionally, more than $40 \%$ of patients with RCC have died of their cancer, in contrast with the $20 \%$ mortality rates associated with prostate and bladder carcinomas. ${ }^{2}$ Overall, 8.9 new cases are diagnosed per 100,000 population per year, with a male-to-female predominance of $3: 2$. There is also an age and racial predilection, with typical presentation in the sixth and seventh decades of life, and a $10 \%$ to $20 \%$ higher incidence of RCC in African Americans for unknown reasons. ${ }^{3}$ Approximately 4\% of all cases are estimated to be familial ${ }^{3}$ however, these cases have yielded the most insight into the molecular mechanisms of RCC.

With the increasing use of imaging, the incidence of RCC has been increasing by an average of $3 \%$ per year for whites and $4 \%$ per year for African Americans. ${ }^{3}$ This has correlated with an improved 5 -year survival rate for patients with small renal masses. ${ }^{4}$ Unfortunately, the prognosis associated with advanced RCC (locally advanced, lymph node involvement or systemic metastases) remains poor. Lymph node involvement has long been recognized as a dire prognostic sign because it is associated with 5 - and 10 -year survival rates of $5 \%$ to $30 \%$ and $0 \%$ to $5 \%$, respectively. ${ }^{5}$ Systemic metastases portend a particularly poor prognosis for RCC, with 1-year survival of less than $50 \%$, 5 -year survival of $5 \%$ to $30 \%$, and 10 -year survival of $0 \%$ to $5 \%{ }^{6}$ 
The options for treatment of patients with advanced RCC have been limited. Studies have shown that cytokine therapy including interferon alpha and interleukin 2 provide a moderate response and limited survival benefit. ${ }^{6}$ Randomized data have suggested that cytoreductive nephrectomy combined with interferon alpha provides a modest survival benefit of 3 months. ${ }^{7}$ Unfortunately, none of these modalities have provided any durable survival advantage for patients with advanced RCC. In light of this, new targeted therapies have been sought.

\section{Molecular mechanisms of RCC}

Several prognostic factors for RCC have been identified including pathologic tumor (TNM) stage, tumor size, tumor grade and histologic subtype. ${ }^{8}$ In particular, conventional clear cell RCC, which represents $85 \%$ of all RCC, has been associated with a poor prognosis. The familial form of the common clear cell variant of RCC occurs in von Hippel-Lindau (VHL) disease. Identification of the VHL (tumor suppressor) gene on chromosome 3 has helped elucidate the molecular mechanisms of clear cell RCC.

The primary function of the VHL protein complex is to target the hypoxia-inducible factor 1 (HIF-1) for ubiquitin-mediated degradation, keeping the levels of HIF-1 low under normal conditions. HIF-1 is an intracellular protein that plays an important role in regulating cellular responses to hypoxia, starvation, and other stresses. Inactivation or mutation of the VHL gene leads to dysregulated expression of HIF-1, and this protein begins accumulating within the cell. ${ }^{9}$ This leads to upregulation of the expression of vascular endothelial growth factor (VEGF), the primary proangiogenic growth factor in clear cell RCC, in addition to platelet derived growth factor (PDGF) and glucose transporter (Glut-1), which are also involved in tumorogenesis (Figure 1). ${ }^{10}$

\section{Targeted therapies for RCC}

With the identification of VEGF and PDGF in the development of clear cell RCC, various therapies targeted at inhibiting angiogenesis have been developed. Two tyrosine kinase inhibitors (TKI) of the VEGF and other pathways, sunitinib and sorafenib, have been shown to improve survival in patients with advanced RCC in phase III randomized controlled trials. In the first-line management of metastatic RCC, sunitinib was found to prolong overall survival (OS) (26.4 vs 21.4 months in the placebo group; $P=0.05$ ) and progression free survival (PFS) (11 vs 5.5 months; $P<0.001$ ) when compared to interferon alpha therapy. ${ }^{11}$ Sorafenib improved PFS (5.5 vs 2.8 months for placebo; $P<0.000001)$ in patients with cytokine refractory metastatic RCC. ${ }^{12}$ These agents have provided a new avenue for treatment for patients with advanced RCC. However, the tumor may become refractory to these treatments over time. As a result, alternative therapies have been sought for these patients.

\section{MTOR inhibitors}

An additional class of targeted agents known as the mammalian target of rapamycin (mTOR) inhibitor has become a central target for cancer therapy, RCC in particular. The mTOR protein kinase is a $289-\mathrm{kDa}$ serine/threonine specific kinase, of which the carboxyl group of TOR is similar to the catalytic domain of PI3-kinase (PI3K). mTOR primarily functions as a key controller of cell proliferation, cell growth, and cell survival. ${ }^{13}$

mTOR is a central regulator of cell growth and proliferation via a mechanism of regulation of translation initiation. ${ }^{14}$ mTOR regulates the translation of ribosomal proteins - two proteins in particular: p70S6K1 and 4E-BP1. mTOR-dependent phosphorylation of ribosomal p70S6 kinase causes translation of ribosomal proteins. Translation is also regulated by phosphorylation of eukaryotic translation initiation factor 4E (eIF4E)-binding protein 1 (4E-BP1). Activation of p70S6 kinase and eIF4E by mTOR is induced by insulin and other growth factors. Therefore, the mTOR pathway controls the translation of mRNA that encode proteins that are required for G1 cell-cycle progression and S-phase initiation. mTOR acts as a gatekeeper for cell-cycle progression, and mTOR inhibition results in prolonged G1 phase or G1 arrest.

Currently, two mTOR inhibitors that have been investigated in phase III trials in the management of metastatic RCC: temsirolimus and everolimus.

\section{Temsirolimus}

Temsirolimus, or CCI-779, a soluble ester analog of rapamycin, was selected for development as an anti-cancer agent based on its prominent anti-tumor profile and favorable pharmaceutical and toxicological characteristics in preclinical studies. Temsirolimus was found to have improved aqueous solubility and stability over rapamycin as an anti-cancer agent.

A phase III trial in poor-risk advanced RCC patients and no prior systemic therapy enrolled 626 patients in an open-label study comparing temsirolimus, interferon alpha and combination temsirolimus/interferon alpha. Patients were randomized in a 1:1:1 fashion to arm 1, interferon 


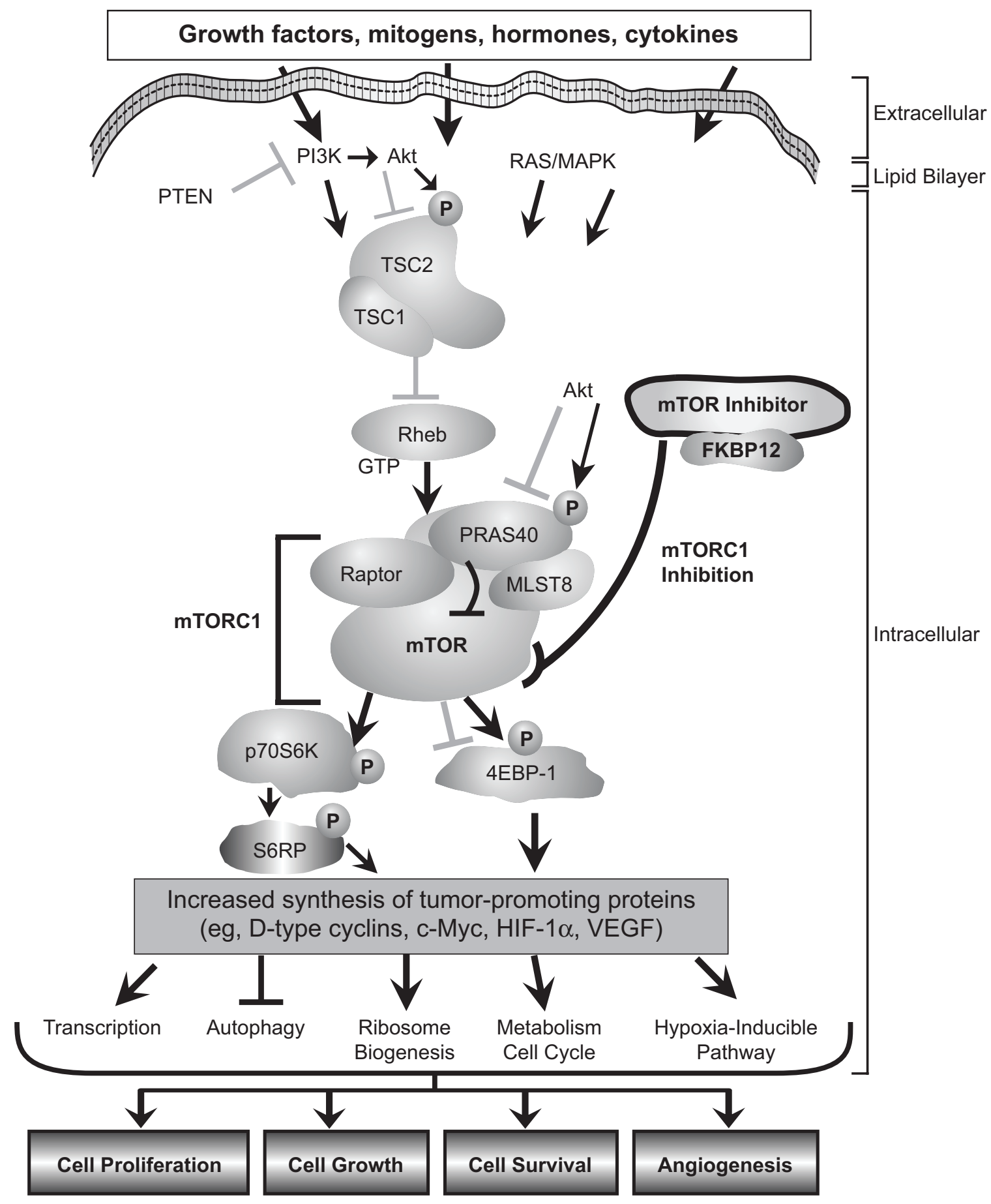

Figure I The mammalian target of rapamycin (mTOR) network. mTOR is a central component of the phosphoinositide 3' kinase/protein kinase B (PI3K)/Akt signaling pathway that mediates eukaryotic cell growth and proliferation. The mTOR kinase is the catalytic component of two distinct multiprotein complexes called mTOR complex I (mTORCI) and mTORC2. mTORCI also contains raptor, MLST8, and praline-rich Akt substrate 40 (PRAS40) (a repressor). In tumor cells, $\mathrm{mTORCI}$ activity is aberrantly elevated. Diverse positive and negative growth signals influence the activity level of $\mathrm{mTORCI}$, many of which converge upon the tuberous sclerosis complex I (TSCI)/TSC2 complex and Ras homolog enriched in brain (Rheb). PI3K receives activating signals from growth factor receptors or it may be activated constitutively in some tumors. Phosphatase and tensin homolog (PTEN) reverses the action of PI3K and functions as a tumor suppressor. Akt also promotes mTORCI activity by phosphorylating TSCI/TSC2 and PRAS40, resulting in guanosine-5'-triphosphate (GTP) loading of Rheb and suppression of the PRAS40 repressor. Downstream, $\mathrm{mTORCI}$ phosphorylates the 70-kDa ribosomal protein S6 kinase (P70S6K) (activation) and eukaryotic translation initiation factor 4E binding protein (4EBP-I) (inhibition of negative regulator) to increase messenger RNA translation of specific proteins. mTOR-regulated proteins include D-type cyclins and c-Myc (which increase cell proliferation), hypoxia-inducible factor-I $\alpha$ (HIF-I $\alpha$ ) (which increases synthesis of the proangiogenic factor vascular endothelial growth factor [VEGF]), various glycolytic enzymes, and growth factors (which can promote cell survival). Activation and inhibition induced by direct phosphorylation at one or more sites is indicated by a phosphate (P). The mTOR inhibitors (rapamycin, temsirolimus, everolimus, deforolimus) inhibit $\mathrm{mTORCl}$ by first binding to the intracellular protein FK506 binding protein IA, I $2 \mathrm{kDa}$ (FKBPI2); the resultant mTOR inhibitor-FKBPI 2 complex then binds mTOR, blocking $\mathrm{mTORCI}$ to inhibit its kinase activity. Through the specific inhibition of mTORCI activity, the mTOR inhibitors display a multifunctional biologic activity profile, blocking cell proliferation, cell growth, cell survival, and angiogenesis in preclinical tumor models. ${ }^{13}$ Figure reprinted with permission from Wyeth Pharmaceuticals, Collegeville, PA, USA. Abbreviations: MAPK, mitogen-activated protein kinase; S6RP, S6 ribosomal protein. 
alpha up to 18 million U subcutaneously 3 times weekly; arm 2, temsirolimus $25 \mathrm{mg}$ intravenously once per week; or arm 3, temsirolimus $15 \mathrm{mg}$ intravenously once per week + interferon alpha 6 million U subcutaneously 3 times weekly. ${ }^{14}$ Of these patients, $67 \%$ had prior nephrectomy. The primary study endpoint was overall survival, and the study was powered to compare the temsirolimus arms with the interferon alpha arm.

Single-agent temsirolimus $(n=209)$ was shown to significantly increase the overall survival (10.9 vs 7.3 months; $P=0.0069)$ of patients with metastatic renal cell carcinoma and poor risk factors, compared with interferon alpha $(\mathrm{n}=207)$. Overall survival by treatment arm was 7.3 months (interferon alpha), 10.9 months (temsirolimus), 8.4 months (temsirolimus/interferon alpha). Median PFS was 1.9 months (interferon alpha), 3.7 months (temsirolimus), 3.7 months (temsirolimus/interferon alpha). Objective response $(\mathrm{CR}+\mathrm{PR})$ were $7 \%(\operatorname{arm} 1), 9 \%(\operatorname{arm} 2)$ and 11\% (arm 3). The authors concluded that single-agent temsirolimus (25 mg intravenously weekly) significantly increases the overall survival of first-line, poor-risk advanced renal cell carcinoma patients compared with interferon alpha, with an acceptable safety profile.

\section{Everolimus}

Everolimus (RAD001) is also a derivative of sirolimus and has both immunosuppressant and antiangiogenic properties. It targets the cellular protein mTOR, a regulator of signaling pathways associated with the abnormal growth, proliferation, and survival of cancer cells. ${ }^{15}$ Recent evidence has added support to the value of everolimus in the treatment of metastatic RCC. ${ }^{16}$

\section{Safety of everolimus in advanced RCC}

In phase I studies of everolimus in a variety of tumors, O'Donnell et al attempted to identify the optimal dosing regimen. ${ }^{17}$ Thirty-seven patients were treated on a weekly regimen with dose escalation. Drug limiting toxicity (DLT) occurred in 1 of 6 patients at $50 \mathrm{mg}$ but none of 4 patients at $70 \mathrm{mg}$. An additional 37 patients were treated on the daily regimen, where DLT occurred in none of 4 patients at $5 \mathrm{mg}$ and in 1 of 6 patients at $10 \mathrm{mg}$. Thus, the higher dosage cohorts were expanded for each regimen. The maximum tolerated dose (MTD) for everolimus was not reached.

The most common drug-related adverse events were rash and erythema, which occurred in 44 patients (48\%). It appeared within the first month in 32 patients, was principally acneiform (34\%) or erythematous (18\%), and occurred most commonly over the upper body and head. The frequency of occurrence increased with increasing dosage in both the daily and weekly treatment groups. Fatigue was observed in 31 patients (34\%). Grade 3 fatigue was observed in only 2 patients, in association with stomatitis in 1 patient and depression in the other; both patients discontinued therapy. It was severe (grade 3 ) in 1 patient (10 mg/day), with severity decreasing to grade 1 after interruption and dose reduction to $5 \mathrm{mg}$ /day. Gastrointestinal (GI) toxicities reported in 61 patients $(66 \%)$ included stomatitis, nausea, vomiting, anorexia, constipation, and abdominal pain or distension. Although GI toxicity was usually mild, stomatitis (erythematous, ulcerative) was grade 3 in 3 patients, 1 of whom discontinued treatment. Hematologic abnormalities were reported in 17 patients. In general, a mostly moderate decrease in platelet and neutrophil counts occurred rapidly after introduction of treatment but remained constant thereafter. Hemoglobin showed a tendency to decline over time, possibly related to the underlying disease.

Severe (grade 3 or 4 ) drug-related adverse events occurred in 19 of 92 patients $(20.6 \%)$, more in the $10 \mathrm{mg}$ daily dosing than any other dosing regimen. Five patients had severe toxicity that was suspected to be drug related, 4 of whom required hospitalization. Hemorrhagic gastritis occurred after 6 days of therapy (10 mg/day) in an 82 year-old man with a history of peptic ulcer and without thrombocytopenia, who recovered after drug discontinuation. Recurrent epistaxis occurred in a patient $(10 \mathrm{mg} /$ day $)$ with moderate thrombocytopenia (platelet count, $97 \times$ $\left.10^{9} / \mathrm{L}\right)$. Bronchiolitis obliterans organizing pneumonia was confirmed histologically in 1 patient ( $70 \mathrm{mg} /$ week) who developed cough and dyspnea after 4 to 6 weeks of therapy, which worsened to grade 3 severity by month 3 but resolved completely after drug discontinuation and glucocorticoid therapy. A patient (10 mg/day) with lung metastases and grade 2 lymphopenia, without neutropenia, developed grade 3 pneumonia that resolved with antibiotic therapy. Finally, grade 3 fatigue and stomatitis developed in another patient after three doses (50 mg/week). There were no suspected drug-associated fatalities. The most common severe toxicities were mucositis, hyperglycemia and neutropenia (3 of 92 for each). Pneumonia represented the only grade 4 toxicity. Hyperlipidemia (hypercholesterolemia and/or hypertriglyceridemia), which was again considered drug related, was reported in 7 patients and was grade 3 (hypertriglyceridemia) in 2 patients, both of whom improved with statin therapy. 
Infections were reported in 41 patients. A relationship to study drug was suspected in only 12 patients, whose infections included cutaneous herpes simplex ( 5 patients), oral candidiasis complicating stomatitis (4 patients), pneumonia ( 2 patients), rhinitis ( 2 patients), conjunctivitis ( 1 patient), influenza-like illness ( 1 patient), and a combination of upper respiratory and urinary tract infections (1 patient).

Phase $\mathrm{II}^{18}$ and phase III $^{16}$ trials have found minimal adverse drug events in patients with advanced RCC treated with everolimus. Common side effects including mouth ulcers and stomatitis (36\% vs $7 \%$ in placebo), asthenia and fatigue ( $28 \%$ vs $20 \%$ ), rash (25\% vs $4 \%$ ), diarrhea (17\% vs $3 \%$ ), anorexia ( $16 \%$ vs $6 \%$ ), nausea ( $15 \%$ vs $8 \%$ ), vomiting ( $12 \%$ vs $4 \%)$, cough $(12 \%$ vs $4 \%)$, peripheral edema $(10 \%$ vs $3 \%$ ), pneumonitis ( $8 \%$ vs $0 \%$ ) and dyspnea ( $8 \%$ vs $2 \%$ ) were seen in both everolimus and placebo groups with a low proportion of grade 3 or 4 events for both groups. Patients receiving everolimus had higher rates of grade 3 or 4 stomatitis, infections, and non-infectious pneumonitis than did those in the placebo group. Treatment discontinuation was required for $28(10 \%)$ patients receiving everolimus (with pneumonitis, dyspnea, lung disorder, and fatigue the most common reasons) and for $5(4 \%)$ patients in the placebo group. Of the 8 patients with grade 3 pneumonitis, 6 discontinued everolimus therapy. Four showed complete clinical resolution, and 3 improved to grade 2 or less. The main laboratory abnormalities included anemia, lymphopenia, thrombocytopenia and neutropenia. Hypercholesterolemia, hypertriglyceridemia, hyperglycemia was higher in the everolimus arm. ${ }^{16}$

\section{Efficacy of everolimus in advanced RCC}

In phase I studies, partial response (PR) was observed in a 71-year-old man (70 mg/week) with RCC and in another patient with RCC, adrenal and lung lesions had shrunk with PR evaluation at $10 \mathrm{mg} /$ day. ${ }^{17}$ Five of the $10 \mathrm{RCC}$ patients remained progression free at 6 months.

A recent phase II trials have looked at the efficacy of everolimus in renal cell carcinoma. Amato et al reported a median PFS of 11.2 months and the median overall survival was 22.1 months in patients with RCC treated with everolimus after cytokine or cytotoxic therapy. ${ }^{18}$ The eligibility criteria included predominant clear cell histology, measurable disease, adequate organ function, absence of central nervous system metastasis, Zubrod performance status of 2 or better and no more than 1 prior treatment. The therapy was everolimus given at $10 \mathrm{mg}$ daily dose with no interruptions on a 28-day cycle. PET-CT was also utilized in addition to RECIST defined criteria for response-evaluation, which was performed at the end of each cycle for 2 cycles. The primary end point was response rate to everolimus utilized in the daily dosing schedule of $10 \mathrm{mg}$ orally. Partial responses were observed in $5(14 \%)$ patients, stable disease lasting at least 3 months was reported in 27 (73\%) patients, and stable disease lasting at least 6 months was reported in $21(57 \%)$ patients.

In a randomized, placebo-controlled phase III trial by Motzer et al 410 patients were randomized 2:1 comparing everolimus to placebo in those patients who had progressed on sunitinib or sorafenib or both. ${ }^{16}$ The primary end-point was PFS, with 290 events to achieve $90 \%$ power. Secondary endpoints were safety, response, patient-reported outcomes, and overall survival. Key eligibility criteria were metastatic RCC with clear cell component, measurable disease, progressive disease on or within 6 months of treatment with sunitinib, sorafenib, or both. Exclusion criteria included prior exposure to mTOR inhibitors (temsirolimus), untreated brain metastasis or uncontrolled co-morbidities. Prior bevacizumab and cytokine treatment was permitted.

Of the 410 patients randomized, 272 patients received everolimus $10 \mathrm{mg}$ daily, and 138 patients received placebo. The median age was 61 years, and 60 years, in the everolimus and placebo arms, respectively. The main sites of metastases were lung $(73 \%, 81 \%)$, bone $(37 \%, 31 \%)$ and liver $(35 \%$, $36 \%$ ). More than $90 \%$ of patients in both arms had greater than 1 site of metastatic disease. $96 \%$ of patients in the everolimus arm and $95 \%$ of patients in the placebo arm had prior nephrectomy. $71 \%$ of patients had been treated with sunitinib, 55\% with sorafenib, and 26\% with sunitinib and sorafenib. Nearly $71 \%$ in the everolimus group and $79 \%$ in the placebo group had progressed during prior therapy and the median length of treatment was 95 days in everolimus and 57 days in placebo respectively. Treatment in both cohorts continued until progression, severe toxicity, death or discontinuation. Randomization was revealed only at progression and cross-over to open-label everolimus was permissible upon progression on placebo.

Patient discontinuation in the everolimus arm was 31\% due to progressive disease, $10 \%$ due to adverse events, $3 \%$ due to death. In the placebo arm, $73 \%$ patients discontinued due to progressive disease, $1 \%$ due to adverse events, and $2 \%$ due to death.

Median PFS was 4.9 months (4 to 5.5 months) for the everolimus arm versus 1.9 months (1.8 to 1.9 months) for control arm $(P<0.0001)$. The best responses observed 
in the two arms were $1 \%$ vs $0 \%$ partial response, $63 \%$ vs $32 \%$ stable disease, $19 \%$ vs $46 \%$ progressive disease favoring everolimus. In $17 \%$ of patients on everolimus and $22 \%$ of patients on placebo, disease could not be assessed. At 6 months, progression-free probability was $26 \%$ in patients receiving everolimus and $2 \%$ in patients receiving placebo. While there was a significant benefit from everolimus, the quality of life data from the original publication did not show any difference between everolimus and placebo. No observable difference was evident between the two groups with respect to the time to clear-cut deterioration of patient-reported outcomes.

Based on these data showing statistically significant improvement in PFS as compared with placebo, everolimus established clinical benefit as a second-line therapy in patients who progress on first-line targeted therapy, including sunitinib and sorafenib. Everolimus can be proposed as the new standard of care in the second-line setting for patients progressing on targeted therapy with VEGF inhibitor.

While evidence is growing about the role of everolimus as a second-line monotherapy for advanced RCC, the combination of everolimus with another agent is a viable option if the second agent targets the signaling in a different pathway. ${ }^{19}$ There is recent evidence that the combination of bevacizumab (a monoclonal antibody to VEGF) with everolimus in patients with advanced RCC (either as first-line therapy or after TKI failure) may be associated with a significant objective response rate $(21 \%)$ and stable disease rate/minor response rate $(69 \%)$. Toxicity when these agents are combined included proteinuria (19\% grade $3-4)$, fatigue ( $9 \%$ grade 3-4), mucositis/stomatitis (49\% grade 3-4), hyperlipidemia (45\% grade $3-4)$, nausea (40\% grade $3-4$ ) and hypertension $(25 \%) .{ }^{20}$ The final results of this study are pending; however, these preliminary results add weight to the concept of multi focal approaches to targeted therapy.

An additional phase I trial combining sunitinib (37.5 mg in the 4 weeks on/2 weeks off schedule) and everolimus (daily or weekly regimen) reported at the 2009 ASCO Annual meeting revealed a significant dose limiting toxicity when the two agents were combined. ${ }^{21}$ The severe adverse events included endocarditis, gastrointestinal hemorrhage with severe anemia and pulmonary embolism. An altered dosing schedule is planned for phase II of this trial.

\section{The role of everolimus in advanced $R C C$}

With the emerging evidence for targeted therapies in the management of advanced RCC, algorithms have been created to help guide best practice for these agents. ${ }^{22}$ In patients with good or intermediate prognosis disease who are treatment naive, sunitinib or bevicizimab with interferon alpha are suggested treatments; however, for patients with poor prognosis, temsirolimus is recommended. Everolimus is best suited for patients with advanced RCC who are refractory to tyrosine kinase inhibitors (TKIs) or other mTOR inhibitors while sorafenib is recommended for patients who are refractory to cytokine therapy.

\section{Conclusions}

Based on these data, everolimus is the first agent to show clinical benefit in a randomized, double-blind study in patients who have progressed on first-line targeted therapy including sunitinib and sorafenib, with improvement in overall PFS compared to placebo. While everolimus has been associated with drug-related toxicity, it appears to be well tolerated in phase II and III trials and appears to be quite safe. Trials combining everolimus with other targeted therapies are pending but do also appear promising from a tolerability perspective.

Sunitinib remains the first-line treatment for patients with advanced RCC. However, the therapeutic benefits of these agents become limited over time. As a result, alternative treatment options are necessary. Phase III trials of everolimus in this patient population have yielded promising results for salvage therapy. Thus, everolimus can be proposed as the new standard of care in second-line setting for those patients progressing on VEGF-inhibitor therapy.

The current frontier of mTOR inhibitors is limited to temsirolimus, an intravenous prodrug, and everolimus, an orally bioavailable agent. The former is used primarily as first-line therapy for poor prognosis patients, whereas everolimus is indicated as second-line therapy for those failing TKIs. Clinical trials for everolimus in the first-line setting as well as in combination with other targeted therapies are ongoing. This agent may yield superior results to TKIs in either primary monotherapy or in the multimodal therapy setting for advanced RCC and provides new hope for the management of one the most lethal genitourinary malignancies.

\section{Disclosures}

The authors declare no conflicts of interest.

\section{References}

1. Landis SH, Murray T, Bolden S, et al. Cancer statistics: 1999. CA Cancer J Clin. 1999;49:8-31.

2. Pantuck AJ, Zisman A, Belldegrun A. Biology of renal cell carcinoma: changing concepts in classification and staging. Semin Urol Oncol. 2001;19(2):72-79. 
3. Chow WH, Devesa SS, Warren JL, et al. Rising incidence of renal cell cancer in the United States. JAMA. 1999;281(17):1628-1631.

4. Kessler O, Mukamel E, Hadar H, et al. Effect of improved diagnosis of renal cell carcinoma on the course of the disease. J Surg Oncol. 1994;57(3):201-204.

5. Phillips CK, Taneja SS. The role of lymphadenectomy in the surgical management of renal cell carcinoma. Urol Oncol. 2004;22(3):214-223.

6. Motzer RJ, Russo P. Systemic therapy for renal cell carcinoma. J Urol. 2000;163(2):408-417.

7. Flanigan RC, Mickisch G, Sylvester R, et al. Cytoreductive nephrectomy in patients with metastatic renal cancer: a combined analysis. J Urol. 2004;171(3):1071-1076.

8. Sorbellini M, Kattan MW, Snyder ME, et al. A postoperative prognostic nomogram predicting recurrence for patients with conventional clear cell renal cell carcinoma. J Urol. 2005;173(1):48-51.

9. Maxwell PH, Wiesener MS, Chang GW, et al. The tumour suppressor protein VHL targets hypoxia-inducible factors for oxygen-dependent proteolysis. Nature. 1999;399(6733):271-275.

10. Maranchie JK, Linehan WM. Genetic disorders and renal cell carcinoma. Urol Clin North Am. 2003;30(1):133-141.

11. Motzer RJ, Hutson TE, Tomczak P, et al. Sunitinib versus interferon alfa in metastatic renal-cell carcinoma. N Engl J Med. 2007;11;356(2): 115-124.

12. Escudier B, Eisen T, Stadler WM, et al. Sorafenib in advanced clear-cell renal-cell carcinoma. N Engl J Med. 2007;11;356(2):125-134.

13. Kapoor A, Figlin RA. Targeted inhibition of mammalian target of rapamycin for the treatment of advanced renal cell carcinoma. Cancer. 2009;115(16):3618-3630.
14. Hudes G, Carducci M, Tomczak P, et al. Temsirolimus, interferon alfa, or both for advanced renal-cell carcinoma. $N$ Engl $J$ Med. 2007;356(22):2271-2281.

15. Kapoor A. Inhibition of mtor in kidney cancer. Curr Oncol. 2009;16 Suppl 1:S33-S39.

16. Motzer RJ, Escudier B, Oudard S, et al. Efficacy of everolimus in advanced renal cell carcinoma: a double-blind, randomised, placebo-controlled phase III trial. Lancet. 2008;372(9637):449-456.

17. O'Donnell A, Faivre S, Burris HA, 3rd, et al. Phase I pharmacokinetic and pharmacodynamic study of the oral mammalian target of rapamycin inhibitor everolimus in patients with advanced solid tumors. $J$ Clin Oncol. 2008;26(10):1588-1595.

18. Amato RJ, Jac J, Giessinger S, et al. A phase 2 study with a daily regimen of the oral mTOR inhibitor RAD001 (everolimus) in patients with metastatic clear cell renal cell cancer. Cancer. 2009;115(11): 2438-2446.

19. Miller RE, Larkin JM. Combination systemic therapy for advanced renal cell carcinoma. Oncologist. 2009;14(12):1218-1224.

20. Whorf RCHJ, Spigel DR, et al. Phase II study of bevacizumab and everolimus (RAD001) in the treatment of advanced renal cell carcinoma (RCC). J Clin Oncol. Proceedings, ASCO. 2008;252s: Abstract 5010.

21. Kroog GS. Phase I trial of RAD001 (everolimus) plus sunitinib in patients with metastatic renal cell carcinoma. J Clin Oncol. 2009;27(15S): Abstract 5037.

22. Bellmunt J, Guix M. The medical management of metastatic renal cell carcinoma: integrating new guidelines and recommendations. BJU Int. 2009;103(5):572-577.
Drug, Healthcare and Patient Safety

\section{Publish your work in this journal}

Drug, Healthcare and Patient Safety is an international, peer-reviewed open-access journal exploring patient safety issues in the healthcare continuum from diagnostic and screening interventions through to treatment, drug therapy and surgery. The journal is characterized by the rapid reporting of reviews, original research, clinical, epidemiological and

\section{Dovepress}

post-marketing surveillance studies, risk management, health literacy and educational programs across all areas of healthcare delivery. The manuscript management system is completely online and includes a very quick and fair peer-review system. Visit http://www.dovepress.com/ testimonials.php to read real quotes from published authors. 